\title{
La tarification de l'eau d'irrigation en Tunisie : une analyse en équilibre général
}

Pricing methods of irrigation water in tunisia: a general equilibrium approach

\section{Chokri Thabet, Louis-Pascal Mahé et Yves Surry}

\section{(2) OpenEdition}

\section{Journals}

\section{Édition électronique}

URL : http://journals.openedition.org/economierurale/3126

DOI : 10.4000/economierurale.3126

ISSN : 2105-2581

\section{Éditeur}

Société Française d'Économie Rurale (SFER)

\section{Édition imprimée}

Date de publication : 5 janvier 2005

Pagination : 51-69

ISSN : 0013-0559

\section{Référence électronique}

Chokri Thabet, Louis-Pascal Mahé et Yves Surry, «La tarification de l'eau d'irrigation en Tunisie : une analyse en équilibre général », Économie rurale [En ligne], 285 | Janvier-février 2005, mis en ligne le 05 janvier 2007, consulté le 30 avril 2019. URL : http://journals.openedition.org/economierurale/3126 ; DOI : 10.4000/economierurale.3126

Ce document a été généré automatiquement le 30 avril 2019.

(c) Tous droits réservés 


\title{
La tarification de l'eau d'irrigation en Tunisie : une analyse en équilibre général
}

Pricing methods of irrigation water in tunisia: a general equilibrium approach

\author{
Chokri Thabet, Louis-Pascal Mahé et Yves Surry
}

\section{NOTE DE L'AUTEUR}

Chokri Thabet remercie vivement MM. M. Ben Said, B. Thabet, L. Lachaal, M. S. Bachta et A. Gohin pour leurs conseils enrichissants et les orientations pertinentes qu'ils ont fait sur ce travail.

\section{Introduction et problématique}

1 Au cours des dix dernières années, la Tunisie a mené une stratégie de développement axée sur l'agriculture, l'industrie manufacturière et le tourisme ${ }^{1}$. Le secteur agricole occupe près de $22 \%$ de la population active (ministère du Développement économique, 1999). L'emploi agricole est dominé par le travail familial qui représente plus de $77 \%$ du total. La part de travail salarié dans le secteur agricole est donc limitée à environ $23 \%$. L'agriculture (y compris l'ensemble des activités liées au secteur) est encore le plus grand utilisateur des ressources du pays : $80 \%$ de l'eau, $90 \%$ des terres utiles et $40 \%$ de la population totale.

2 Les produits des grandes cultures constituent la quasi-totalité des importations. La Tunisie exporte principalement des fruits (des dattes surtout, et des agrumes dans une moindre mesure) et des produits de la pêche, qui représentent à eux deux près de $80 \%$ des exportations agricoles totales. L'activité agroalimentaire est dominée par la 
production de viande ( $20 \%$ du total de la production de la branche), la production d'huile et de corps gras (19\%) et la transformation des grains (18\%). La Tunisie importe principalement des huiles végétales $(29 \%$ du total des importations de produits agroalimentaires), des sucres ( $21 \%)$ et des produits laitiers (18\%). Les principales exportations agroalimentaires sont l'huile d'olive (52\%) et les produits de conserve ( $24 \%$ ). Le secteur irrigué occupe 7,3\% de la superficie agricole utile du pays et sa part dans la production agricole au cours des derniers plans de développement économique et social est passée de 29 à $33 \%$. Le secteur irrigué contribue aussi pour $20 \%$ dans la valeur des exportations agricoles.

3 Bien que le taux de croissance du Produit national brut (PNB) ait été en moyenne de $5 \%$ durant les années 1990, ses fluctuations ont été très sensibles, principalement à cause de la variabilité de la production agricole (due particulièrement aux aléas de pluviosité). La dépendance de la production agricole par rapport à la pluviométrie a suscité des efforts considérables d'investissements hydrauliques ${ }^{2}$. Néanmoins, l'augmentation de l'offre d'eau n'a pas été accompagnée de mesures de rationalisation visant à maîtriser une demande sans cesse croissante. La politique adoptée était donc centrée sur la promotion de l'offre par un investissement croissant, afin de rattraper une demande galopante.

Les prix de l'eau d'irrigation ont été longtemps très faibles et déconnectés de la réalité des coûts (ministère de l'Agriculture, 1997). Les agriculteurs des périmètres irrigués bénéficiaient de subventions explicites et implicites. Les subventions explicites étaient justifiées par la recherche de la sécurité alimentaire, l'amélioration du solde du commerce extérieur et la réduction de l'exode rural. Dans le cadre du Plan d'ajustement structurel agricole (PASA), ces subventions ont été réduites graduellement et ne représentent plus qu'un faible pourcentage du prix de l'eau d'irrigation. Cependant, les subventions implicites représentent encore une part importante du prix payé par les agriculteurs, dans la mesure où ce prix n'inclut pas les coûts d'investissement dans l'infrastructure hydraulique. D'après la Banque mondiale (1995), les prix de l'eau payés par les différents usagers en 1994 sont de 0,04 dinar tunisien par mètre cube $\left(\mathrm{DT} / \mathrm{m}^{3}\right)^{3}$ pour les agriculteurs, $0,32 \mathrm{DT} / \mathrm{m}^{3}$ pour les ménages et $0,59 \mathrm{DT} / \mathrm{m}^{3}$ pour les industries et le tourisme. Toutefois, les coûts de production varient de 0,15 à $0,52 \mathrm{DT} / \mathrm{m}^{3}$ pour l'eau d'irrigation, 0,45 à $0,86 \mathrm{DT} / \mathrm{m}^{3}$ pour les ménages et près de $0,45 \mathrm{DT} / \mathrm{m}^{3}$ pour l'usage industriel et touristique. La faiblesse des tarifs de l'eau d'irrigation a incité les utilisateurs à considérer la ressource en eau comme étant un « don du ciel ", alors qu'elle est un bien rare, au même titre que les autres facteurs de production. Il s'en est suivi des gaspillages de la ressource en eau ainsi que des subventions importantes qui grèvent le budget de l'État.

5 Plusieurs études ont souligné la gravité du problème et la nécessité d'agir pour gérer la demande en eau par les différents utilisateurs (ministère de l'Agriculture, 1995 et 1996). Selon le Plan Bleu sur l'avenir du bassin méditerranéen, la Tunisie est classée parmi les pays où la pression sur la ressource en eau est forte avec un indice d'exploitation ${ }^{4}$ des ressources naturelles et renouvelables se situant à $58 \%$ (Benblidia et al, 1998). Les ressources en eau disponibles par personne étaient d'environ $540 \mathrm{~m}^{3}$ par an en 1990 , comparées à $1195 \mathrm{~m}^{3}$ par an au Maroc. Selon les estimations du Plan Bleu reportées dans Margat (1992), en 2025, les ressources en eau par habitant seront de $310 \mathrm{~m}^{3} /$ an en Tunisie et de $600 \mathrm{~m}^{3} / \mathrm{an}$ au Maroc. Pour les deux pays, cet indicateur serait inférieur à $1000 \mathrm{~m}^{3}$ / an, seuil jugé critique par différents experts et signe de pénurie chronique. Sur la base de ce concept, la Tunisie est considérée comme un pays où la rareté des ressources en eau 
risque de poser une contrainte sérieuse au développement de son économie. Ce constat est confirmé par Margat et Treyer (2004). Il devient donc évident qu'une gestion durable de cette ressource devrait être fondée sur une approche intégrée visant une meilleure prise en compte des coûts de la ressource par la demande.

Pour faire face à cette situation, le gouvernement tunisien a lancé plusieurs programmes d'économie d'eau reposant sur trois composantes interdépendantes : i) conservation de la ressource en eau (réduction des pertes et fuites techniques, etc.) ii) réforme de la tarification de l'eau d'irrigation et iii) réforme du cadre institutionnel dans le sens de la décentralisation (incitations à la création d'Associations d'intérêts collectifs). Nous examinons ici uniquement la deuxième composante du programme, relative à la réforme de la politique de prix de l'eau d'irrigation.

7 Il est communément admis que la tarification de l'eau d'irrigation est un outil puissant permettant d'infléchir sa demande afin qu'elle s'ajuste à l'offre (Johansson et al., 2002). Cependant, les méthodes de tarification de l'eau d'irrigation présentent des propriétés incitatives et allocatives différentes (Tsur et Dinar, 1995). Quelle est alors la méthode la plus appropriée qui soit économiquement efficace et socialement équitable? Une ressource est allouée d'une manière optimale quand son prix reflète son coût d'opportunité. Dans ce contexte, une tarification au coût marginal est généralement la méthode la plus recommandée par la théorie économique, car elle permet une allocation optimale de premier rang de la ressource (Hirshleifer et al., 1960). Toutefois, l'importance des coûts fixes associés à la construction des barrages et à l'infrastructure de distribution a amené Dinar et Subramanian (1997), Thoban (1997) et Dinar (2002) à se demander si la tarification au coût marginal ne devrait pas être abandonnée au profit de méthodes de tarification de second rang.

Le déficit public du «budget de l'eau » n'étant pas soutenable à long terme, l'argument central de ces économistes repose sur la remise en question de la tarification des services publics au coût marginal en raison des asymétries d'information et du coût marginal non nul des fonds publics.

En Tunisie, les pouvoirs publics visent à court terme le recouvrement des frais variables de maintenance et de fonctionnement de l'infrastructure hydraulique et, à plus long terme, la couverture des coûts fixes des investissements consentis dans ce domaine (ministère de l'Agriculture, 1997). Pour ce faire, la méthode de tarification binôme " personnalisée ${ }^{5}$ semble être retenue. Ce tarif comporte deux parties : la première est fixe et basée sur la surface de terre irriguée alors que la seconde est variable selon le volume d'eau consommé. Les droits fixes par hectare irrigué sont destinés à couvrir les coûts fixes, et les sommes proportionnelles au volume consommée, à couvrir les coûts marginaux de production d'eau. Sans chercher à déterminer la tarification optimale, nous comparons en termes d'efficacité et d'équité trois méthodes de tarification de second rang, à savoir : i) une tarification au coût moyen ii) une tarification binôme "classique " composée d'un tarif proportionnel à la consommation volumétrique en eau et un abonnement fixe et iii) une tarification binôme "personnalisée » ${ }^{6}$. Par efficacité économique, on entend le niveau de richesse agrégée maximal généré par les ressources disponibles en eau (ici le solde des effets de bien-être et de transferts) alors que, l'analyse d'équité se rapporte aux effets de répartition sur les revenus des ménages de différentes tarifications : ici des changements relatifs de bien-être des ruraux par rapport à celui des urbains. 
10 On examine aussi l'impact de la réforme de la tarification de l'eau d'irrigation sur ses usages alternatifs sachant qu'initialement l'agriculture consomme $80 \%$ des ressources en eau, les ménages $13 \%$, les industries $5 \%$ et le tourisme $2 \%$. Cette analyse prend en compte la mobilité et la substitution des facteurs primaires de production et l'allocation résultante des ressources entre secteurs. Les réactions des productions agricoles et des industries de transformation sont aussi examinées.

11 Pour répondre à ces objectifs, nous développons un modèle d'équilibre général calculable (EGC) pour l'économie tunisienne. Ce choix méthodologique est motivé par notre souci de prendre en considération les mécanismes d'interdépendance générale et les effets de rétroaction des décisions des agents sur la structure de la production et de la demande ${ }^{7}$. De plus, comparée à d'autres approches (analyse coûts-bénéfices, modèles d'équilibre partiel), l'utilisation d'un modèle EGC présente l'avantage de prendre en compte les changements dans certains secteurs de l'économie autres que ceux affectés directement par la politique en question.

12 Les quelques modèles EGC qui ont été développés jusqu'à maintenant pour étudier les politiques de gestion de l'eau d'irrigation dans les pays du Moyen-Orient et d'Afrique du Nord diffèrent entre eux à plusieurs niveaux. Ainsi, Goldin et Roland-Holst (1994) supposent l'existence d'une offre d'eau parfaitement élastique, pouvant répondre à toute demande. Löfgren (1996) fait l'hypothèse de l'existence d'une quantité globale d'eau fixe prévalant dans l'économie alors que Decaluwé et al. (1998) modélisent explicitement la production d'eau avec différentes technologies selon que l'eau est extraite des barrages ou de nappes souterraines. Toutefois, ils ne distinguent pas de secteur de production et de distribution d'eau potable. Autrement dit, l'eau provenant des barrages ou des nappes souterraines pourrait être directement utilisée pour la consommation humaine sans traitement spécifique. Du côté de la demande, certains modèles considèrent uniquement la demande agricole en eau (Löfgren, 1996; Goldin et Roland-Holst, 1994), alors que Decaluwé et al. (1998) distinguent trois types de demande (agricole, industrielle et domestique). Le modèle EGC utilisé dans ce travail suppose l'existence d'une quantité globale d'eau fixe et prend en compte l'ensemble des utilisateurs d'eau (agricoles, ménages, industries et services).

13 L'article ${ }^{8}$ comporte trois sections. La première présente les principales caractéristiques du modèle EGC utilisé ainsi que la représentation du «marché » de l'eau. La deuxième décrit les scénarios examinés et les résultats obtenus. La dernière section conclut en présentant les limites.

\section{Modélisation des secteurs agricoles et du marché de l'eaude l'économie tunisienne}

14 Les principales caractéristiques du modèle d'équilibre général calculable (EGC) de la Tunisie sont présentées dans l'encadré ci-après. Bien que comparable à la majorité des modèles EGC développés pour l'économie de ce pays 9 , il possède néanmoins une double particularité, à savoir une représentation appropriée des processus de production en agriculture et une modélisation explicite du marché de l'eau ${ }^{10}$.

Une bonne représentation des technologies de production agricole nécessite une désagrégation " adéquate » des facteurs primaires de production. Une des particularités de notre modèle est de distinguer le travail salarié du travail non salarié et la terre 
irriguée de la terre en sec. La première est importante dans le contexte tunisien du fait de la dominance du travail familial ( $77 \%$ du total, $c f$. introduction). La distinction entre terre irriguée et terre en sec est liée à la différence de qualité entre ces deux types de terre. En effet, une terre équipée en infrastructure d'irrigation se prête à plusieurs usages et donne des rendements plus élevés qu'une terre en sec et de ce fait, procure à son propriétaire une rente plus élevée. Cette distinction nous permettra aussi de modéliser explicitement une tarification binôme personnalisée.

Principales caractéristiques du modèle EGC construit pour la Tunisie

- Nature du modèle. Le modèle en question est statique et assez détaillé pour simuler les implications de la réforme de la politique de l'eau sur les secteurs agricoles et non agricoles.

- Taille du modèle. 35 secteurs dont 12 agricoles et 8 agroalimentaires. Les secteurs agricoles sont répartis en trois sous-groupes : le premier comprenant la production de blé tendre, blé dur, autres céréales, fourrages, autres fruits et autres cultures, utilise les deux catégories de terre terres en sec et en irrigué ; le deuxième sous-groupe considère la production en terre irriguée de betterave à sucre, agrumes, dattes et légumes; le dernier secteur agricole produit des olives sur des «terres en sec». Le modèle prend en compte six catégories de facteurs primaires comprenant la terre en sec et la terre irriguée, le capital, la ressource en eau, le travail salarié et le travail non salarié. Enfin, deux types de ménages, les ménages urbains et ruraux, sont représentés dans le modèle.

- Production. Les technologies relatives à chaque secteur sont mono-produits et à rendements constants, et représentées par des fonctions CES (Constant Elasticity of Substitution) emboîtées. Chaque secteur produit un bien composite qui a pour débouchés le marché intérieur et l'exportation. On suppose la concurrence parfaite et le plein-emploi des facteurs.

- Préférences des consommateurs. Une structure séparable à trois niveaux est adoptée pour représenter les préférences des ménages. Au premier niveau, les ménages allouent leur revenu entre dépenses de consommation de biens et de services, et épargne en supposant une propension marginale à consommer constante. Au deuxième stade, un système linéaire de dépenses (SLD) est spécifié pour expliquer l'allocation des dépenses de consommation entre six postes de consommation, dont quatre agrégés (produits agricoles et de pêche, produits des industries agroalimentaires, reste des produits industriels, reste des services, énergie et eau distribuée). Enfin, à la dernière étape, une formulation de type CES est adoptée pour décrire les décisions de répartition des quatre postes agrégés de consommation.

- Commerce extérieur. Il est spécifié d'une façon standard en adoptant l'hypothèse d'Armington (1969). Ainsi, les produits d'origines intérieure et importée sont considérés comme des substituts imparfaits et sont modélisés selon une fonction CES. De même, il existe une distinction entre les produits offerts sur le marché intérieur et ceux destinés à l'exportation. Ceci permet d'obtenir des fonctions d'offre des produits intérieurs et exportés à partir des conditions d'optimalité associées à une fonction de transformation de type CET (Constant Elasticity of Transformation) entre les produits domestiques et exportés. Par ailleurs, on suppose que la Tunisie est un petit pays à l'exportation et à l'importation, impliquant en conséquence que les prix mondiaux sont exogènes au modèle. 
- Fermeture du modèle. La dotation de chaque facteur primaire de production est fixe. Les facteurs «capital » et «travail » sont mobiles entre les divers secteurs de l'économie. Bien que la terre soit spécifique à l'agriculture, cela n'empêche pas ce facteur d'être mobile entre ses secteurs irrigués et non irrigués. Le déficit public (ou l'épargne) est endogène et les dépenses publiques fixées à leurs niveaux de l'année de base (1992). Le taux de change est flexible et induit l'équilibre de la balance des paiements. La règle de bouclage adoptée est néoclassique, permettant l'équilibre entre l'épargne et l'investissement, et respectant la loi de Walras.

- Calibrage du modèle. Le modèle est calibré à partir de l'adaptation à nos besoins d'une matrice de comptabilité sociale (MCS) construite pour l'année 1992 par Chemingui et Dessus (1999). Cette dernière a été complétée par des informations tirées des Comptes de la Nation, d'annuaires de statistiques agricoles, de budgets économiques et du travail doctoral de Kress (1994). Les valeurs retenues pour les paramètres du modèle (élasticités) s'inspirent des travaux de Chemingui et Dessus (op. cit.) pour tout ce qui touche aux secteurs de production et aux échanges commerciaux. Quant aux élasticités-revenu des six biens de consommation, nous avons utilisé les estimations effectuées par Ayadi et al. (1997) pour les ménages ruraux et urbains en Tunisie. Pour plus de détails sur la construction de la MCS et de la sélection des paramètres du modèle, voir Thabet (2003).

L'annexe I (in fine) illustre le processus de production adopté pour les secteurs agricoles utilisant les deux catégories de terre. La structure arborescente sous-jacente révèle l'existence d'un processus de décision à étages où les producteurs agricoles combinent dans un premier temps, selon une fonction CES, les engrais à l'eau d'irrigation. Cet agrégat "eau-engrais " sera ensuite combiné dans une CES, à un deuxième niveau au facteur " terre agrégée », lui-même issu d'une combinaison de terres irriguée et pluviale selon une fonction CES. L'agrégat ainsi obtenu est ensuite combiné à un autre intrant composite liant, par le biais d'une fonction CES, le capital, le travail salarié et le travail non salarié pour former à un troisième niveau une sorte de valeur ajoutée "élargie ". Celle-ci constituée des facteurs primaires et des engrais est utilisée dans des proportions fixes avec le reste des inputs intermédiaires pour définir la production agricole.

L'adoption de cette spécification a certains avantages, mais aussi des caractéristiques bien spécifiques qu'il faut expliquer plus en détail. Tout d'abord, en supposant l'utilisation d'intrants intermédiaires dans des proportions fixes, le problème de résolution de l'équilibre général sera beaucoup plus simplifié et par conséquent, on réduit la complexité des calculs (Ballard et al., 1985). Ensuite, la formulation CES que nous avons adoptée est fort pratique car, tout en restant simple à manipuler, elle est globalement valide $^{11}$ et répond à toutes les caractéristiques requises en termes de régularité. De plus, elle permet de reproduire toute la gamme de comportements depuis la stricte complémentarité jusqu'à la substitution parfaite, en passant par l'élasticité de substitution unitaire caractéristique de la fonction Cobb-Douglas. Cette formulation présente toutefois un inconvénient: lorsque ses composantes ne sont pas des substituts parfaits (élasticité de substitution finie et différente de zéro), la fonction n'est additive qu'en valeurs et non en quantités ${ }^{12}$.

Pour ce qui est des activités agricoles qui utilisent uniquement la terre irriguée ou la terre en sec, nous avons conservé le même type de technologie que pour les secteurs utilisant les deux catégories de terre, tout en éliminant toutefois la fonction CES qui les liait. Une telle spécification de la technologie agricole prend en compte d'une part, les travaux 
antérieurs de modélisation sur la question (Edwards et al, 1996 et Hexem et Heady, 1978) qui privilégient la nécessité de représenter aussi fidèlement que possible les substitutions entre l'eau d'irrigation et les autres intrants et d'autre part, les caractéristiques propres des processus de production agricole en Tunisie. Cette dernière dimension explique en partie, la spécification adoptée qui peut différer de celles prévalant dans d'autres modèles EGC qui ont abordé ce sujet (Decaluwé et al., 1998 ; Goldin et Roland-Holst, 1994 ; Berck et al., 1991). En se fondant sur les travaux de Belhajhassine (op. cit.), portant sur les cultures irriguées de la région du Cap Bon, nous avons supposé que l'eau d'irrigation est substituable aux engrais dans le cas des activités de production à forte demande en eau et complémentaire pour le reste. De plus, à l'étage combinant la terre à l'agrégat « eauengrais » par une fonction CES, on prend en compte la logique agronomique qui suggère une certaine substitution entre ces deux groupes de facteurs.

Quant au fonctionnement du marché de l'eau, nous supposons que l'État détient une quantité d'eau " primaire » fixe. Cette offre exogène devrait satisfaire la demande d'eau d'irrigation par les secteurs agricoles et celle provenant du secteur de production d'eau potable (SONEDE) ${ }^{13}$. Ce dernier transformera l'eau primaire en eau potable selon un processus de production conventionnel. L'eau primaire est utilisée en proportions fixes avec un facteur primaire composite formé de travail salarié et de capital. L'ensemble de ces facteurs qui forment la valeur ajoutée du secteur est utilisé en proportions fixes avec les consommations intermédiaires (énergie, produits chimiques, etc.) pour produire une eau potable. Cette dernière est destinée aux ménages et aux autres secteurs non agricoles (industries, services et tourisme) de l'économie tunisienne. On fait l'hypothèse que l'eau d'irrigation est subventionnée à un niveau donné (année de base) et que son prix est fixé. En revanche, le secteur eau potable achète à l'État l'eau primaire à un prix qui sera donc déterminé d'une manière endogène de façon à équilibrer l'offre globale d'eau à la demande globale exprimée par toute l'économie. 
Figure 1. Situation initiale de la demande d'eau d'irrigation

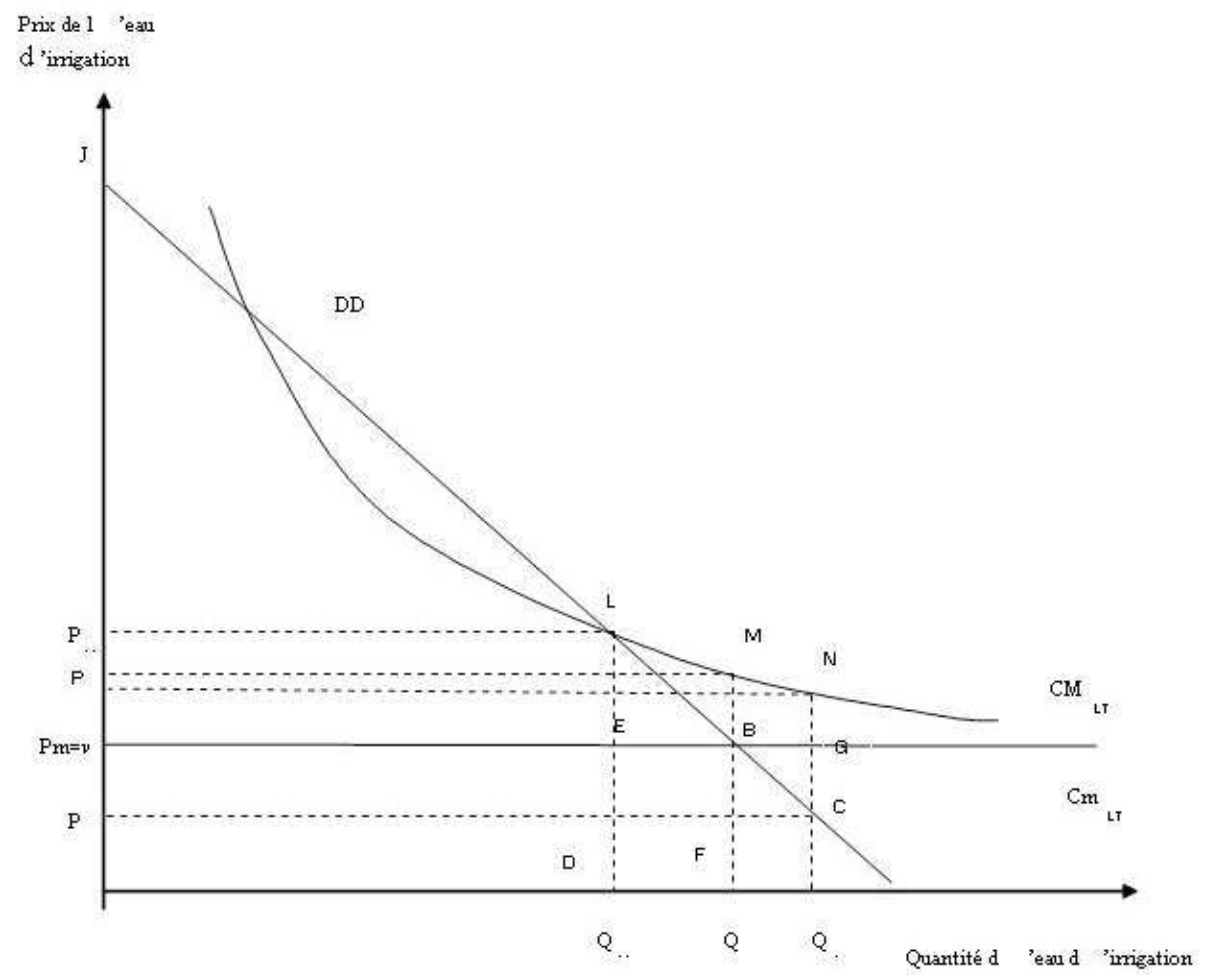

\section{Analyse et discussions de scénarios}

La situation initiale de la demande agricole en eau d'irrigation et de la subvention qui lui est accordée est illustrée par la figure 1. Le coût de fourniture de l'eau comporte une partie fixe A qui correspond à l'infrastructure collective et une partie variable supposée ici proportionnelle au volume d'eau. Le coût marginal $n$ est constant, d'où la droite correspondante $\mathrm{Cm}_{\mathrm{LT}}$.

(1) $C(Q)=A+n \times Q$

(2) $[\mathrm{C}(Q) / Q-n] Q=A$

Le coût moyen $C(Q) / Q$ est donc égal à $A / Q+n$. L'expression (2) montre que la courbe de coût moyen $C M_{L T}$ est une hyperbole équilatère en prenant l'axe des abscisses passant par $P_{m}=n$, dont tous points tels que $L, M$ et $N$ vérifient (2). Le coût marginal $n$ permet le recouvrement des frais d'entretien et de maintenance de l'infrastructure hydraulique ou encore l'ensemble des charges variables associées au fonctionnement du réseau de distribution de l'eau d'irrigation. Lors de l'année de base, les agriculteurs payent un prix de l'eau d'irrigation égal à $P_{0}$ (inférieur à $n$ ) et consomment donc $Q_{0}$. Ils sont alors subventionnés à hauteur de $C\left(Q_{0}\right) / Q_{0}-P_{0}=C_{0}-P_{0}$ qui représente l'écart entre le coût moyen de production d'eau (à la quantité $Q 0$ ) et le prix réel pratiqué. Pour couvrir le coût marginal de la production et distribution de l'eau d'irrigation $n=P_{m}$, l'augmentation du prix de l'eau facturée à atteindre est $P_{m}-P_{0}$.

Le point $B$ est théoriquement le plus efficient pour la société, tout au moins dans la mesure où les coûts fixes $A$ peuvent être couverts par des impôts levés de façon 
forfaitaire, c'est-à-dire sans effet d'allocation sur les ressources productives dans l'économie. Dans ce cas, les usagers paient $\frac{P_{m} \times Q_{M}}{2}$ pour la consommation d'eau et le budget de l'État doit financer le déficit, c'est-à-dire le coût fixe $A$ qui est égal au rectangle $P_{m} B M P$, par la relation (2). Les inefficacités de la tarification actuelle de l'eau d'irrigation et d'une tarification au coût moyen peuvent être identifiées sur la figure 1 . Au prix $P_{0}$, la consommation totale d'eau d'irrigation est $Q_{0}$. À ce prix, les usagers obtiennent un gain de surplus par rapport au point optimal $B$, équivalent à la surface $d u$ trapèze $P_{m} B C P_{O}$. Les coûts budgétaires additionnels par rapport au coût fixe de la subvention unitaire associés à cette situation sont donnés par le rectangle ${ }^{P_{m}} G C P_{0}$. En effet, les coûts fixes sont couverts comme au point $B$ car ils sont égaux au produit $Q_{0} \times\left(C_{0}-P_{0}\right)=$ aire $C_{0} N G P_{m}=Q_{m} \times\left(P-P_{m}\right)=$ aire $P_{m} B M P$. Ainsi, la perte sèche pour la société (ou encore charge morte, égale au solde des transferts) engendrée par une tarification au prix ${ }^{P_{0}}$ est représentée par le triangle BGC.

La tarification au coût moyen (TCM) conduit à un équilibre au point $L$ où la courbe de coût moyen coupe la demande. En effet au prix $P_{M}$, la demande est bien $Q_{M}$ et, par construction, le coût total de la fourniture d'eau est couvert entièrement par l'usager : $P_{M} \times Q_{M}=C\left(Q_{M}\right)=A+n \times Q_{M}$, c'est-à-dire par l'imposition d'une charge $P_{M}$ proportionnelle à la quantité. En passant de $B$ en $L$, les usagers perdent un surplus égal au trapèze $P_{M L} L B P_{m}$. Les contribuables gagnent le coût fixe $A=$ aire $P_{m} B M P=$ aire $P_{M M} L E P_{m}$. En passant de $B$ en $L$ la perte sèche est donc égale au triangle $L E B$. On observe bien que la situation $B$ est plus efficace que les deux autres.

Pour résumer, partant de la situation actuelle, une tarification au coût marginal engendrerait une diminution de la demande d'eau d'irrigation jusqu'au niveau $Q_{m}$ et par suite, entrâne un gain collectif en annulant la perte sèche représentée par le triangle $B G C$, tandis qu'une tarification au coût moyen, permettrait une plus grande économie d'eau d'une valeur de $Q_{M}-Q_{0}$, tout en imputant aux usagers irrigants l'ensemble des coûts associés à la production et la distribution de l'eau d'irrigation. Cependant, la tarification au coût moyen engendre une perte sèche pour la société équivalente au triangle $L E B$, par rapport à la tarification au coût marginal.

21 La difficulté de mettre en œuvre la tarification au coût marginal pose divers problèmes révélés par les travaux récents sur les services publics. Il est en particulier difficile de circonvenir les questions d'asymétrie d'information et d'éviter la charge morte liée au prélèvement d'impôt. Certains travaux (ex. Ballard et al., 1985) ont montré que le coût marginal des fonds publics pouvait être très élevé (jusqu'à $30 \%$ ). Ceci jette des doutes quant à l'optimalité pratique de la tarification au coût marginal accompagnée du financement par l'impôt des coûts fixes. La tarification dite Ramsey-Boiteux (ex. Laffont, 1985) a inspiré la recherche de solutions praticables et plus efficaces quand les coûts de mise en œuvre sont pris en compte. C'est l'objectif des tarifications non linéaires comme les tarifs binômes ou polynômes. Une forme simple concerne le tarif binôme comportant un abonnement fixe calculé de façon à couvrir les coûts fixes et une charge variable égale au coût marginal.

Dans le cas présent, on considère deux cas : la tarification binôme classique $(T B C)$ où l'abonnement est fixe et forfaitaire et la tarification binôme personnalisée (TBP) où l'abonnement est calculé sur une référence individuelle basée sur le nombre d'hectares irrigués. Dans le cas de la TBP, le fait de faire peser la contribution sur la terre qui est 
disponible en quantité fixée (offre inélastique) permet de répartir le financement du service dans l'esprit de Ramsey-Boiteux, dans la mesure où l'on anticipe que l'offre d'eau brute à l'irrigation a une élasticité plus forte que celle de la terre.

Ainsi la charge morte totale du financement sera plus faible. Ceci est illustré sur la figure 2 inspirée ${ }^{14}$ de Laffont (1985) et transposée aux demandes dérivées des facteurs terre et eau. Sur le marché foncier à gauche (où l'on prend le cas extrême d'une offre complètement rigide), le prélèvement de la somme $a \times S_{0}$ égale à l'aire marquée $A$ n'entraîne aucune charge morte puisque l'offre de terres ne peut réagir. En réalité tout le poids de la "taxe » sur la terre sera porté par les propriétaires fonciers qui ne recevront plus qu'un prix diminué de la charge $a$ par hectare. Il n'y a pas de perte allocative liée à ce prélèvement. Par contre sur le marché de l'eau à droite où l'offre d'eau est plus élastique, une réduction du prélèvement sur le même usager passant par une baisse du prix de l'eau de $P_{M}$ à $P_{m}$ (apportant une baisse de contribution des usagers par cette voie de $\left(P_{M}-P_{m}\right) \times Q_{M}=A$, donc égale au prélèvement introduit sur le foncier) va entraîner une annulation de la charge morte liée à la tarification au coût moyen et donner donc une solution globalement plus efficace ${ }^{15}$. Ainsi la TBP sera Pareto supérieure à la TCM . En réalité, l'offre de terre irriguée réagit quand même un peu et il y a une petite charge morte liée à la charge sur le foncier. Mais le bilan du passage de TCM à TBP pourra être positif. L'idée de la tarification binôme classique est la même, mais elle supposait, de façon optimiste, que l'on puisse introduire dans le modèle un prélèvement forfaitaire sans perturber les marchés. Un abonnement identique par sous-secteur agricole donnait des résultats peu réalistes. On en est venu à répartir cette charge en fonction de la valeur ajoutée. Mais alors il s'est avéré que les demandes de facteurs primaires ont réagi et donné des résultats différents de ceux de la TBP basée sur le foncier, et à première vue dans un sens paradoxal.

Les scénarios de simulation portent sur la comparaison, à la situation de départ illustrée par le point $C$ (de la figure 1) des effets en termes d'efficacité et d'équité des trois méthodes de tarification suivantes :

- une tarification binôme "classique " (augmentation du prix de l'eau d'irrigation au niveau $P_{m}$ avec une charge fixe forfaitaire différenciée selon la contribution de chacune des cultures irriguées dans la valeur ajoutée globale de l'agriculture irriguée);

- une tarification binôme " personnalisée » (augmentation du prix de l'eau d'irrigation au niveau $P_{m}$ avec une charge fixe uniforme par hectare irrigué) et ;

- une tarification au coût moyen.

Ces méthodes permettent toutes les trois d'atteindre l'équilibre budgétaire du gouvernement, par le recouvrement des coûts fixes et des coûts variables associés à la production et distribution de l'eau d'irrigation. Néanmoins, nous simulons uniquement une couverture publique de $30 \%$ des coûts fixes ${ }^{16}$. 
Figure 2. Principe de la tarification binôme réduisant la charge morte liée au financement des coûts fixes

\section{2a. Marché foncier et} demande de terres

a

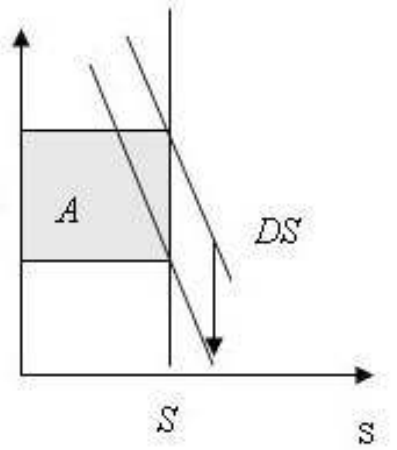

\author{
2b. Marché de l'eau \\ brute et demande \\ d'eal
}

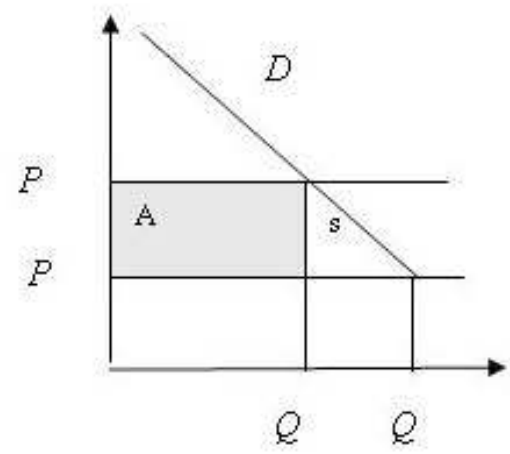

\section{Effets sur le bien-être et analyse en termes d'équité}

Les effets sont résumés dans le tableau 1. Les variations de bien-être des ménages relatifs à la situation actuelle où l'eau d'irrigation est fortement subventionnée sont mesurées par les variations compensatrices qui indiquent les sommes minimales que les ménages devraient recevoir pour accepter l'application de tel ou tel mode de tarification. Pour les ruraux le bien-être diminue, car les variations sont négatives pour les trois nouveaux modes de tarification (TCM : $-36,6 \mathrm{MD}, T B P:-54,5 \mathrm{MD}$ et $T B C:-26,4 \mathrm{MD})^{17}$. Le bien-être des ménages urbains se détériore aussi sauf dans le cas d'une tarification binôme personnalisée (TCM : $-14,7 \mathrm{MD}, \mathrm{TBP}:+20,1 \mathrm{MD}$ et $T B C:-12,2 \mathrm{MD})$. En prenant en considération les effets positifs des trois méthodes de tarification sur le budget du gouvernement dont l'épargne augmente, le gain de bien-être collectif est positif pour une tarification binôme personnalisée et classique. Ce dernier atteint respectivement $-15,9 \mathrm{MD},+3,4 \mathrm{MD}$ et $+0,4 \mathrm{MD}$ dans le cas d'une tarification au coût moyen, binôme personnalisé et binôme classique.

Tableau 1. Variations de bien-être des groupes sociaux et gain collectif avec les nouvelles tarifications par rapport au statu quo (en millions de dinars)

\begin{tabular}{|l|l|l|l|l|}
\hline $\begin{array}{l}\text { Mode de } \\
\text { tarification }\end{array}$ & $\begin{array}{l}\text { Ménages } \\
\text { ruraux }\end{array}$ & $\begin{array}{l}\text { Ménages } \\
\text { urbains }\end{array}$ & $\begin{array}{l}\text { Épargne publique/ } \\
\text { Contribuables }\end{array}$ & $\begin{array}{l}\text { Surplus } \\
\text { global }\end{array}$ \\
\hline TCM & $-36,6$ & $-14,7$ & $+35,4$ & $-15,9$ \\
\hline TBP & $-54,5$ & $+20,1$ & $+37,8$ & $+3,4$ \\
\hline TBC & $-26,4$ & $-12,2$ & $+39,0$ & $+0,4$ \\
\hline
\end{tabular}

Les différences d'impact sur le bien-être résultent des effets sur les prix des produits mais aussi sur les revenus des ménages. Une tarification binôme personnalisée a peu d'effets 
sur les prix des produits mais, engendre une réduction importante de la rente foncière (variable explicite dans la formation des revenus des ménages ruraux), au profit de l'État. Les deux autres tarifications ont des effets plus prononcés sur les prix des produits agricoles et alimentaires et moins forts sur la rente foncière. Sachant que les consommations minimales définies dans le système SLD en produits agricoles et agroalimentaires sont significatives, les revenus excédentaires se trouvent fortement diminués suite à l'augmentation des prix de la majorité des produits agricoles et agroalimentaires. L'importante diminution du prix de l'eau potable à la consommation finale n'arrive pas à compenser l'augmentation des prix des produits agricoles et agroalimentaires. Le bien-être des ménages urbains augmente dans le cas d'une tarification binôme personnalisée à cause de la hausse des prix du travail salarié et du capital (effet revenu), et d'une faible augmentation des prix des produits agricoles et alimentaires.

Si l'on définit comme neutre en terme d'équité un changement proportionnel du bienêtre des ménages ruraux et des ménages urbains, la tarification binôme personnalisée est défavorable à cet égard car elle induit la plus forte perte des ménages ruraux et est la seule qui bénéficie aux ménages urbains. La méthode la plus neutre dans les effets de répartition est la tarification binôme classique. Si l'on suppose qu'à la marge les ménages urbains contribuent pour au moins la moitié des impôts, alors ils sont clairement gagnants dans tous les cas. Ceci peut poser un réel problème d'équité dans la mesure où les revenus urbains sont, en moyenne, plus élevés que les ruraux. La dimension politique d'une réforme de la tarification de l'eau apparaît ainsi clairement. En outre, le gain en terme d'efficacité globale et donc de PNB à redistribuer est faible en regard de l'ampleur des effets de répartition au détriment des ruraux. Et pourtant la sous-tarification de l'eau est source de nombreux gaspillages et les impôts nécessaires à couvrir le déficit du service de l'eau sont générateurs de charge morte incomplètement prises en compte dans la modélisation. Si l'on estimait en outre que la baisse de bien-être des ménages ruraux devrait être accompagnée de mesures de compensation ciblées sur les moins favorisés de ceux-ci, il faudrait aussi les financer sur fonds publics, ce qui atténuerait le gain collectif final. Ceci remet-il en cause l'opportunité d'une réforme de la tarification? Il nous semble que non. En effet, rapportées au revenu sectoriel, les variations compensatrices sont assez modestes (de -1 à $-2 \%$ pour les ruraux et de $-0,1$ à $+0,2 \%$ pour les urbains). Il est permis de penser qu'une réforme avec une application programmée sur quelques années serait à la fois supportable et avantageuse pour l'économie tunisienne.

Le tableau 1 révèle aussi un résultat paradoxal : le bilan de la TBC est moins bon que la TBP qui prend le foncier irrigué comme assiette de prélèvement complémentaire. Or on avait présenté la $T B C$ comme un financement des coûts fixes par abonnement forfaitaire qui devrait n'avoir que des effets revenus et pas ou très peu d'effets réels d'allocation et donc de charge morte. Le paradoxe n'est en fait qu'apparent car, pour des raisons techniques de mise en œuvre, c'est la valeur ajoutée des sous-secteurs agricoles qui a été prise pour assiette de l'abonnement "forfaitaire ». Il s'avère que ce prélèvement a des effets sensibles sur les demandes de facteurs primaires et des effets sur les marchés non négligeables (voir le point suivant, Effets sur les prix). Ainsi notre tarification binôme classique n'est pas aussi forfaitaire que nous l'aurions souhaitée et il serait plus précis de l'appeler « tarification binôme semi-classique ». primaire inélastique montre assez bien la difficulté de réformer le financement des 
services collectifs quand on prend en compte les aspects opérationnels concrets, les contraintes politiques et l'équité. Elle suggère aussi qu'une réforme, annoncée clairement mais appliquée progressivement, est possible et apporte des gains d'efficacité à l'économie tout entière.

\section{Effets sur les prix et la mobilité intersectorielle des facteurs primaires}

Les prix des facteurs primaires de production les plus affectés sont ceux de l'eau d'irrigation, l'eau primaire utilisée par la SONEDE, la terre irriguée et la terre en sec ( tableau 2). Les prix du capital et du travail salarié n'ont varié que marginalement car ils sont déterminés essentiellement par les secteurs non agricoles. En revanche, la terre irriguée et la terre en sec sont spécifiques aux sous-secteurs agricoles. Leurs prix sont plus affectés.

Tableau 2. Effets réels sur l'emploi et effets prix des trois tarifications pour quelques facteurs utilisés dans l'agriculture tunisienne

\begin{tabular}{|l|l|l|l|l|l|l|}
\hline & \multicolumn{4}{|l|}{ Emploi (\%) } & \multicolumn{2}{l|}{ Prix (\%) } \\
\hline Mode de tarification & TCM & TBP & TBC & TCM & TBP & TBC \\
\hline Travail salarié & $-0,34$ & $-0,65$ & $-1,64$ & $+0,02$ & $+0,38$ & $+0,17$ \\
\hline Capital & $-0,35$ & $-0,50$ & $-1,24$ & $-0,16$ & $+0,20$ & $+0,10$ \\
\hline Terre irriguée & & & & $-17,70$ & $-40,40$ & $-10,80$ \\
\hline \hline Eau d'irrigation & $-23,90$ & $-3,00$ & $-3,00$ & $+54,10$ & $+10,50$ & $+10,50$ \\
\hline Engrais & $+7,36$ & $+6,90$ & $+8,30$ & $-0,32$ & $-0,10$ & $-0,39$ \\
\hline
\end{tabular}

31 La couverture par les pouvoirs publics de $30 \%$ des coûts de l'infrastructure hydraulique nécessite une augmentation du prix du $\mathrm{m}^{3}$ d'eau d'irrigation de $+54,1 \%$ dans le cas d'une tarification au coût moyen et de $+10,5 \%$ dans le cas du binôme personnalisé et classique. La charge fixe par hectare irrigué est évaluée à 243 DT dans l'option TBP, alors qu'avec la $T B C$, l'abonnement est variable selon la contribution de chacune des cultures irriguées dans la valeur ajoutée globale de l'agriculture irriguée.

L'augmentation du prix de l'eau d'irrigation pour les secteurs agricoles est accompagnée de diminutions du prix de l'eau « brute » à destination de la SONEDE de - 66,4 \%, - 75,9\% et $-76,9 \%$ dans les trois scénarios successifs. La taille du secteur de l'eau potable qui consomme moins de $20 \%$ des ressources en eau du pays explique la forte chute du prix de l'eau « brute ».

L'effet le plus saillant des trois modes de tarification sur les prix des facteurs primaires de production concerne leurs impacts sur la valeur locative de la terre irriguée. Cette dernière diminue respectivement de $-17,7 \% ;-40,4 \%$ et $-10,8 \%$ dans le cas d'une tarification au coût moyen, binôme personnalisé et binôme classique. Pour gagner sur 
l'acceptation politique de la réforme il faut donc prendre en compte ses effets négatifs sur la valeur de la terre irriguée.

Le mode de faire-valoir prépondérant dans l'agriculture tunisienne est direct (91\%), le métayage et la location ne représentent que $9 \%$ (ministère de l'Agriculture, 1996). Néanmoins dans la région de $\mathrm{Nabeul}^{18}, 22 \%$ des terres agrumicoles sont gérées en métayage ${ }^{19}$ et/ou fermage. A priori, une telle réforme serait à l'avantage des métayers, car ils ne participent qu'avec leurs forces de travail dans le processus de production et verront les prix des produits augmenter alors que les fermiers et les propriétaires doivent payer la redevance ou l'abonnement. Une législation spécifique devrait définir la part des paiements de chacun (propriétaire ou locataire) dans le financement de l'infrastructure hydraulique.

En termes de mobilité intersectorielle de l'eau, la demande agricole diminue de - 23,9\% dans le cas d'une tarification au coût moyen et seulement de $-3 \%$ dans le cas d'un binôme personnalisé et classique. Ainsi, si l'objectif des pouvoirs publics consiste à faire contribuer les agriculteurs au financement des investissements et des équipements hydrauliques existants et à assurer une importante économie d'eau, la méthode de tarification au coût moyen semble être l'outil le plus approprié. Mais les simulations montrent qu'en terme collectif la meilleure solution reste la TBP.

\section{Impacts sur les variables d'équilibre ressources-emplois des principaux produits}


du prix du travail non salarié $(-0,2 \%)$ qui compensent la hausse des prix des autres facteurs de production et en particulier l'eau d'irrigation (+ 10,5\%).

Les prix des exportations tunisiennes (voir annexe II) exprimés en monnaie nationale ( $P E_{i}$ ) sont supposés être exogènes et ne varient qu'en fonction du taux de change, en raison de l'hypothèse de petit pays adopté pour la Tunisie. Les augmentations des prix domestiques $\left(P D_{i}\right)$ sont les plus fortes pour une tarification binôme classique suivie de celle au coût moyen et binôme personnalisé. Il s'ensuit un effet négatif sur les exportations dans le même ordre. Les exportations d'agrumes, de dattes et de légumes accusent des diminutions respectives de $-9,2 \%,-15,2 \%$ et $-8,3 \%$ dans le cas d'une tarification binôme classique contre $-4,8 \%,-9,9 \%$ et $-6,3 \%$ dans le cas d'une tarification au coût moyen et seulement de $-0,3 \%,-0,9 \%$ et $-0,3 \%$ lorsqu'il s'agit d'une tarification binôme personnalisée. Sachant que le secteur irrigué contribue à $20 \%$ des exportations agricoles tunisiennes, et que la politique agricole tunisienne accorde une grande importance au développement des exportations, la tarification binôme personnalisée pourrait être privilégiée par rapport à ce critère.

41 Pour les produits agroalimentaires, à part la production d'huile qui connaît une légère augmentation dans le cas des trois scénarios, tous les autres secteurs agroalimentaires enregistrent des baisses de leur volume de production. La production totale en volume des conserves diminue respectivement de $-2,4 \%,-0,7 \%$ et $-2,9 \%$ dans le cas d'une tarification au coût moyen, binôme personnalisé et binôme classique. Celle du sucre connaît une diminution moins forte de $-1,7 \%,-0,7 \%$ et $-0,9 \%$. Ces résultats s'expliquent principalement par la hausse des prix des produits agricoles et notamment ceux de la betterave à sucre et des légumes, matières premières pour la production de sucre et de conserves.

Tableau 3. Effets réels et effets prix des trois tarifications sur quelques productions agricoles irriguées

\begin{tabular}{|l|l|l|l|l|l|l|}
\hline Productions et prix & \multicolumn{4}{l|}{ Production totale (en \%) } & \multicolumn{3}{l}{ Prix à la production (en \%) } \\
\hline Mode de tarification & TCM & TBP & TBC & TCM & TBP & TBC \\
\hline Blé dur & $+0,50$ & $+0,05$ & $+0,77$ & $-0,64$ & $-0,16$ & $-0,72$ \\
\hline Agrumes & $-1,98$ & $-0,42$ & $-3,71$ & $+2,11$ & $-0,03$ & $+4,41$ \\
\hline Betterave & $-1,78$ & $-0,66$ & $-0,92$ & $+9,41$ & $-0,40$ & $+4,95$ \\
\hline Dattes & $-5,87$ & $-0,77$ & $-8,91$ & $+3,29$ & $+0,21$ & $+5,46$ \\
\hline Autres fruits & $-2,15$ & $-0,40$ & $-2,91$ & $+2,69$ & $-0,08$ & $+4,10$ \\
\hline Légumes & $-2,29$ & $-0,52$ & $-2,81$ & $+3,09$ & $-0,12$ & $+4,39$ \\
\hline \hline Sucre & $-1,70$ & $-0,69$ & $-0,88$ & $+0,60$ & $+0,07$ & $+0,21$ \\
\hline
\end{tabular}

L'importante diminution du prix de l'eau primaire résultant du transfert de cette ressource des secteurs agricoles vers le reste de l'économie induit une augmentation de sa 
demande par la SONEDE. En raison de la technologie de type Léontief adoptée pour ce secteur, on assiste alors à une augmentation de la production finale d'eau potable du même montant, c'est-à-dire de $+10,3 \%,+20 \%$ et de $+20,2 \%$ respectivement dans le cas d'une tarification au coût moyen, binôme personnalisé et binôme classique. La réduction du coût de production de l'eau potable se traduit par d'importantes diminutions de son prix à la consommation finale (respectivement de $-15,4 \%,-26 \%$ et $-26,3 \%$ dans le cas d'une tarification au coût moyen, binôme personnalisé et binôme classique). Cette baisse du prix de consommation de l'eau potable profite aux ménages urbains et ruraux et aux autres secteurs industriels et de services. Il en résulte une augmentation de leur demande en eau potable respectivement de $+11,4 \%,+14,9 \%,+3,2 \%$ et $+0,7 \%$ dans le cas d'une tarification au coût moyen, $+22,6 \%,+28,6 \%,+3,6 \%$ et $+1,6 \%$ pour le binôme personnalisé et de $+22,4 \%,+31,2 \%,+4,3 \%$ et $+1,5 \%$ pour le binôme classique.

La production d'engrais augmente dans les trois scénarios du fait de l'hypothèse d'une substitution significative entre ce facteur et l'eau d'irrigation dans la technologie de production des cultures fortement consommatrices en eau d'irrigation (légumes, agrumes, betterave à sucre et dattes).

Signalons enfin que plusieurs tests de sensibilité ont été effectués sur le modèle. Le premier porte sur les élasticités de substitution dans les secteurs agricoles et en particulier la substitution eau-engrais, le deuxième examine l'effet d'une imparfaite mobilité du travail non salarié entre le secteur agricole et le reste de l'économie et le dernier sur la fixité du stock d'eau, remplacé par une parfaite élasticité de son offre. Les résultats montrent que le modèle est sensible à la relation eau-engrais et assez robuste pour les deux autres tests ${ }^{21}$.

\section{Conclusion}

Le modèle que nous avons discuté repose sur plusieurs hypothèses qui concernent le niveau de désagrégation de l'agriculture tunisienne, les technologies de production des secteurs agricoles et en l'occurrence la relation eau-engrais, le fonctionnement du marché de l'eau et les valeurs de certains paramètres de comportement. Les résultats discutés doivent donc être interprétés dans le cadre général de la réglementation des monopoles naturels. En intégrant plusieurs faits stylisés de la Tunisie nous avons simulé trois modes de tarification de l'eau d'irrigation. Les résultats montrent que, si les pouvoirs publics ont pour objectif de faire contribuer les agriculteurs au financement de l'infrastructure hydraulique sans entraver l'irrigation, ni affecter la balance commerciale agricole et alimentaire, la tarification binôme personnalisée est l'instrument le plus adapté. Si par contre, l'objectif d'économie d'eau est primordial, la tarification au coût moyen semble être la plus appropriée. En termes d'équité, les trois méthodes de tarification présentent des effets négatifs sur le bien-être des ménages ruraux et la tarification binôme personnalisée est la seule à engendrer des impacts positifs sur le bienêtre des ménages urbains. Ce qui renseigne sur un écart plus important entre les revenus des ménages ruraux et urbains. Mais en rapport aux revenus sectoriels, ces pertes de bien-être restent faibles et inférieures à $2 \%$ pour les ruraux. La tarification binôme classique s'est avérée manquer de réalisme mais la tarification binôme personnalisée ouvre une voie de réforme raisonnable. 
Globalement, les résultats montrent qu'à long terme, l'agriculture tunisienne serait capable d'absorber les effets d'une réforme de la tarification de l'eau d'irrigation. Les principales raisons vont de la capacité des producteurs agricoles à ajuster leurs combinaisons factorielles (liées bien sûr aux spécifications adoptées pour représenter les technologies agricoles irriguées et en sec), à l'inélasticité de la demande domestique des produits agricoles et agroalimentaires (les offres des produits sont déterminées par les niveaux des demandes en vertu de l'hypothèse des rendements d'échelle constants). L'hypothèse de plein-emploi des ressources en eau explique aussi la faiblesse des effets relatifs de la réforme de la tarification de l'eau d'irrigation. En effet, les impacts négatifs sur l'agriculture irriguée d'une hausse du prix de l'eau sont compensés par des gains des ménages consommateurs d'une eau potable désormais plus abondante. Cet effet est d'autant plus grand que les ménages sont peu désagrégés : ce que le ménage rural perd en tant que producteur est compensé parce qu'il gagne en tant que consommateur. Le résultat serait différent si les ménages ruraux étaient à leur tour désagrégés, certains, spécialisés en cultures irriguées, subissant de plein fouet la hausse du tarif, sans possibilité de compensation. Ce qui nous ramène dans une perspective future à désagréger les ménages ruraux selon leurs occupations professionnelles.

D'autres limites du modèle pourraient faire l'objet de travaux de recherche ultérieurs. À cet égard, considérer les producteurs agricoles comme des ménages "producteurconsommateur » et développer une modélisation appropriée du comportement de ces agents apparaîtrait judicieux (Löfgren et Robinson, 1999). Le modèle est purement statique et n'inclut pas de comportements propres d'investissement des agents économiques. Cette composante permettrait la prise en compte de la création de nouvelles ressources en eau. Il serait aussi possible de réduire le niveau de désagrégation de l'économie tunisienne et de centrer l'analyse sur les secteurs les plus concernés par la réforme.

\section{BIBLIOGRAPHIE}

Ayadi M., Krishnakumar J., Matoussi M.-S. Combining Spatial and Temporal Variations in the Estimation of Price Elasticities for Tunisian Households.Actes du Colloque de la Septième Conférence Internationale sur l'utilisation des données de panel, Paris, 19 et 20 juin 1997.

Armington P.-S. A Theory of Demand for Products Distinguished by Place of Production. IMF Staff Papers, 1969, vol. 16, p. 159-178.

Ballard Charles L., Fullerton D., Shoven J.-B., Whalley J. A General Equilibrium Model for Tax Policy Evaluation. Chicago, University of Chicago Press, 1985.

Banque mondiale. Republic of Tunisia : Towards the 21st Century.Country Economic Memorandium, Washington, October 1995.

Belhajhassine N. Tarification de l'eau et agriculture irriguée : le cas de la Tunisie. Thèse de Doctorat, Université des Sciences Sociales de Toulouse, 1997.

Benblidia M., Margat J., Vallée D. Pénuries d'eau prochaines en Méditerranée. Futuribles, 1998. 
Berck A., Robinson S., Goldman G. The Use of Computable General Equilibrium Models to Assess Water Policies. In Dinar and D. Zilberman (Eds), “The Economic and Management of Water and Drainage in Agriculture”, Boston : Kluwer Academic Publishers, 1991.

Burniaux J.-M. Le radeau de la méduse : Analyse des dilemmes alimentaires. Economica, Paris, 1987.

Chemingui M.-A., Dessus S. La libéralisation de l'agriculture tunisienne et l'Union européenne : une vue prospective. Centre de développement de l'OCDE. Documents techniques, 1999, nº 144.

Cockburn J., Decaluwé B., Dostie B. Concurrence imparfaite, économies d'échelle et commerce extérieur en Tunisie : une aventure ambiguë. In B. Decaluwé, A. Martens et L. Savard (Eds), « La politique économique du développement et les modèles d'équilibre général calculable », Presses de l'Université de Montréal, Montréal, 2001, chapitre 13.

Decaluwé B., Patry A., Savard L. Quand l'eau n'est plus un don du ciel : MEGC appliqué au Maroc. Revue Économique du Développement. 1998, vol. 3-4, p. 149-187.

Dinar A. "Guidelines for Pricing Irrigation Water Based on efficiency, Implementation Cost and Equity Consideration”. World Bank Final Project Report, forthcoming, 2002.

Dinar A., Subramanian A. Water prices experiences, an international perspective. World Bank, technical paper, $1997, n^{\circ} 386$.

Edwards B., Howitt K.R., Flaim S. Fuel, Crop and Water Substitution in Irrigated Agriculture. Resource and Energy Economics, 1996, vol. 18, p. 311-331.

Goldin I., Roland-Holst D. Economic Policies for Sustainable Resource Use in Marocco. In I. Goldin and L.-A. Winters (Eds), “The Economics of Sustainable Development”, New York. Cambridge University Press, 1994.

Hexem R.-W., Heady E.-O. Water Production Function for Irrigated Agriculture. Ames : The Iowa state University Press, 1978.

Hirshleifer, J.-J.-C, De Haven and J.-W. Milliman. Water Supply : Economics, Technology and Policy. Chicago, Illinois : The University of Chicago Press, 1960.

Johansson R.-C., Tsur Y., Roe T.-L., Doukkali R., Dinar A. Pricing Irrigation Water : a review of theory and practice. Water Policy, 2002, 4 (2), p. 173-199.

Kress D.-H. Trade Liberalization and Employment in Tunisia : a general equilibrium analysis with increasing returns to scale and imperfect competition. Phd, University of North Carolina, Chapell Hill, USA, 1994.

Laffont J.-J. Cours de théorie microéconomique. Volume I, Fondements de l'économie publique, Economica, Paris, 1985.

Laffont J.-J., Tirole J. A theory of incentives in procurement and Regulation. MIT press, Cambridge, 1993.

Löfgren H. The Cost of Managing with Less : Cutting Water Subsidies and Supplies in Egypt's Agriculture. In Pfeifer Karen (Ed.), "Research in Middle East Economics", Greenwich, Connecticut, JAI Press 1996, vol. 1, p. 83-107.

Löfgren H., Robinson S. Non Separable Farm Household Decisions in a Computable General Equilibrium Model. American Journal of Agricultural Economics, 1999, vol 81(3), p. 663-673.

Margat J., Treyer S. L'eau des Méditerranéens : Situation et Perspectives. Plan Bleu, Sophia Antipolis, 2004. 
Margat J. Prospective des besoins et des ressources en eau des pays africains riverains de la Méditerranée : contributions du Plan Bleu. Revue Canadienne d'Études du développement, 1992, numéro spécial.

Matoussi M.-S. Gestion durable de la demande en eau : la seule alternative capable de relever les défis menaçants. $8^{\mathrm{e}}$ conférence annuelle, Economic Research Forum, Caire, 2002.

Ministère de l'Agriculture. Perspectives de l'agriculture tunisienne et mutations internationales. Diagnostic et perspectives. Étude réalisée par COMETE Engineering, 1995. - Étude sur la Stratégie des Ressources Naturelles. Rapport principal établi par SCET-TUNISIE et BDPA-SCETAGRI pour la Direction Générale de la Planification, du Développement et des Investissements Agricoles, 1996. - Étude de la gestion et de la Tarification de l'eau d'irrigation au niveau des périmètres irrigués : Diagnostic de la gestion actuelle. Étude réalisée par le CNEA et BRL Ingénierie, 1997. - EAU 21 : stratégie du secteur de l'eau en Tunisie, à long terme, 2030. Rapport final, 1998. - Étude du Secteur de l'Eau. Direction Générale des Ressources en Eau, Tunisie, 1999.

Ministère du Développement Économique. Résultats de l'enquête nationale sur l'emploi de 1997. Institut National de la Statistique, 1999, septembre.

Rogers P. The Agenda for the Next Thirty Years. In Peter Rogers, Peter Lydon (Eds.), "Water in the Arab World : Perspectives and Prognoses", The American University in Cairo Press, Cairo, 1994.

Sghaier M. Tarification et allocation optimale de l'eau d'irrigation dans les systèmes de production de la région oasienne de Nefzaoua (Sud de la Tunisie). Thèse de Doctorat en sciences biologiques appliquées, section agronomie, Université de Gent, 1995.

Thabet C. Réforme de la politique des prix de l'eau d'irrigation en Tunisie : approche en équilibre général. Thèse de doctorat, ENSA de Rennes, juillet 2003.

Thoban M. Formal Water Markets : Why, When, and How to introduce tradable water rights. The World Bank Research Observer, 1997, 12(2), p. 161-179.

Treyer S. Analyse des stratégies et prospectives de l'eau en Tunisie. Plan Bleu, Sophia Antipolis, 2002.

Tsur Y. et Dinar A. Efficiency and Equity Considerations in Pricing and Allocating Irrigation Water. The World Bank, Policy Research, 1995, Working Paper, 1460.

\section{ANNEXES}

Annexe I. Représentation de la technologie de production des secteurs agricoles 


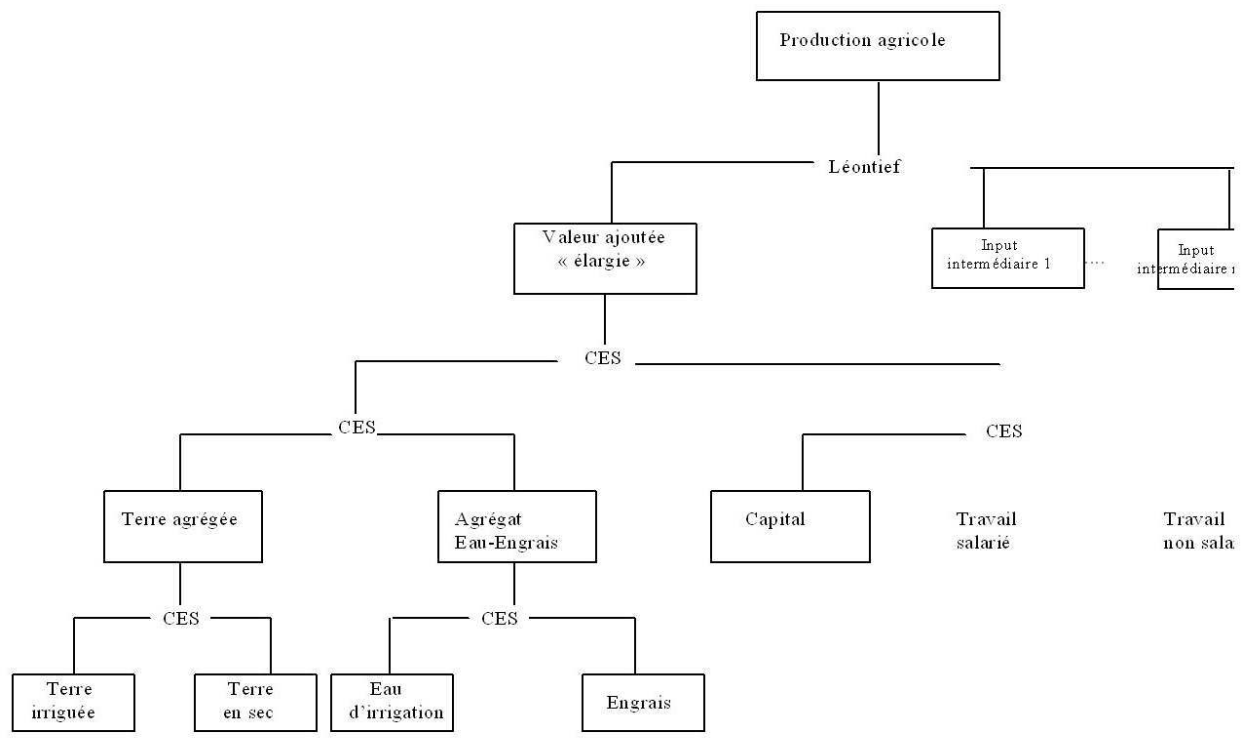

Annexe II. Effets sur les variables d'équilibre ressources-emplois des principaux produits

\begin{tabular}{|c|c|c|c|c|c|c|c|c|c|c|c|}
\hline & Produits & $\begin{array}{l}\text { Production } \\
\text { totale }\left(\mathrm{Y}_{\mathrm{i}}\right)\end{array}$ & $\begin{array}{l}\text { Prix à la } \\
\text { production } \\
\left(\mathrm{P}_{\mathrm{i}}\right)\end{array}$ & $\begin{array}{l}\text { Importations } \\
\left(\mathrm{YM}_{\mathrm{i}}\right)\end{array}$ & $\begin{array}{l}\text { Exportations } \\
\left(\mathrm{YE}_{\mathrm{i}}\right)\end{array}$ & $\begin{array}{l}\text { Production } \\
\text { dom. }\left(\mathrm{YD}_{\mathrm{i}}\right)\end{array}$ & $\begin{array}{l}\text { Prix } \\
\text { domestique } \\
\left(\mathrm{PD}_{\mathrm{i}}\right)\end{array}$ & & & & \\
\hline & & $\begin{array}{l}\text { (variation } \\
\text { en } \% \text { ) }\end{array}$ & $\begin{array}{l}\text { (variation } \\
\text { en } \% \text { ) }\end{array}$ & $\begin{array}{l}\text { (variation } \\
\text { en } \% \text { ) }\end{array}$ & $\begin{array}{l}\text { (variation } \\
\text { en } \% \text { ) }\end{array}$ & $\begin{array}{l}\text { (variation } \\
\text { en } \% \text { ) }\end{array}$ & $\begin{array}{l}\text { (variation } \\
\text { en } \% \text { ) }\end{array}$ & & & & \\
\hline & TCM & TBP & $T B C$ & TCM & $T B P$ & $T B C$ & $T C M$ & TBP & $T B C$ & $T C M$ & TBP \\
\hline Blé dur & $+0,50$ & $+0,05$ & $+0,77$ & $-0,64$ & $-0,16$ & $-0,72$ & $+0,08$ & $-0,15$ & $+0,28$ & - & - \\
\hline Betterave & $-1,78$ & $-0,66$ & $-0,92$ & $+9,41$ & $-0,40$ & $+4,95$ & - & - & - & - & - \\
\hline Olives & $+0,28$ & $+0,09$ & $+0,50$ & $-0,33$ & $-0,05$ & $-0,37$ & - & - & - & $+0,49$ & $+0,24$ \\
\hline Agrumes & $-1,98$ & $-0,42$ & $-3,71$ & $+2,11$ & $-0,03$ & $+4,41$ & - & - & - & $-4,82$ & $-0,29$ \\
\hline Dattes & $-5,87$ & $-0,77$ & $-8,91$ & $+3,29$ & $+0,21$ & $+5,46$ & - & - & - & $-9,94$ & $-0,94$ \\
\hline $\begin{array}{l}\text { Autres } \\
\text { fruits }\end{array}$ & $-2,15$ & $-0,40$ & $-2,91$ & $+2,69$ & $-0,08$ & $+4,10$ & $+7,78$ & $-0,91$ & $+12,13$ & $-5,67$ & $-0,21$ \\
\hline Légumes & $-2,29$ & $-0,52$ & $-2,81$ & $+3,09$ & $-0,12$ & $+4,39$ & $-0,32$ & $-0,63$ & $-0,07$ & $-6,30$ & $-0,27$ \\
\hline Huiles & $+0,33$ & $+0,11$ & $+0,57$ & $-0,31$ & $-0,02$ & $-0,33$ & $-0,40$ & $-0,35$ & $-0,25$ & $+0,73$ & $+0,36$ \\
\hline Sucre & $-1,70$ & $-0,69$ & $-0,88$ & $+0,60$ & $+0,07$ & $+0,21$ & $+0,65$ & $-0,68$ & $+0,32$ & $-3,94$ & $-0,70$ \\
\hline
\end{tabular}




\begin{tabular}{|l|l|l|l|l|l|l|l|l|l|}
\hline SONEDE & $+10,25$ & $+20,02$ & $+20,23$ & $-15,44$ & $-26,01$ & $-26,31$ & - & - \\
\hline Engrais & $+7,36$ & $+6,90$ & $+8,30$ & $-0,32$ & $-0,10$ & $-0,39$ & $+5,81$ & $+5,10$ \\
\hline
\end{tabular}

SONEDE signifie la quantité d'eau produite et distribuée par la SONEDE aux ménages et aux différents secteurs industriels et de services de l'économie tunisienne.

\section{NOTES}

1. Ces trois secteurs contribuaient respectivement à $14,4 \%, 14,6 \%$ et $3,9 \%$ du produit intérieur brut en 1995.

2. . La part des investissements hydrauliques dans l'investissement agricole total a souvent été proche de $60 \%$.

3. DT : dinar tunisien qui équivalait en 1994 à un dollar US.

4. . L'indice d'exploitation désigne les quantités d'eau prélevées en pourcentage du total théorique des ressources en eau renouvelables moyennes. Toutefois, cet indicateur ne tient pas compte des pénuries locales ou conjoncturelles au sein d'un même pays. Cet indice s'élève à $33 \%$ en Algérie, $39 \%$ au Maroc et $93 \%$ en Egypte.

5. Cette dénomination a été utilisée par Laffont et Tirole (1993) pour désigner le fait que la couverture des coûts fixes supportés par le monopole naturel se fasse par une taxe fixe liée à l'utilisation d'un facteur de production.

6. . Notons que notre modèle ne prend pas en compte les coûts additionnels liés à la mise en $œ u v r e$ de ces politiques. Nous supposons que les structures institutionnelles existantes peuvent assurer la mise en place des différents modes de tarification sans coûts supplémentaires significatifs.

7. . Notons que dans le passé, plusieurs approches ont été utilisées pour étudier la gestion des ressources en eau en Tunisie et plus généralement dans la région du Moyen-Orient et de l'Afrique du Nord. Les études centrées sur l'évaluation de la tarification de l'eau d'irrigation en Tunisie ont été menées dans un cadre d'équilibre partiel ou à l'échelle d'exploitations agricoles. Sans être exhaustif, on peut citer Sghaier (1995), Belhajhassine (1997), Matoussi (2002) et Rogers (1994). Peu d'études centrées sur l'eau d'irrigation utilisent des modèles d'équilibre général calculables (EGC) qui, dans tous les cas, sont statiques. Pour une revue de littérature sur la question, voir Johansson et al. (2002).

8. . Cet article est issu d'une communication faites aux Premières journées européennes des thèses en économie et gestion agricole, agroalimentaire et rurale, organisées à Montpellier les 11 et 12 juin 2003 par la SFER en collaboration avec l'AIEA2 et les UMR Moïsa, Lameta et Innovation. L'analyse a bénéficié du soutien financier de la coopération franco-tunisienne (projet CMCU entre l'INAT et l'ENSAR) de l'INRA et du laboratoire d'économie rurale de l'INRAT.

9. Il existe une longue lignée de modèles EGC de la Tunisie qui ont été développés en adoptant un cadre théorique concurrentiel et walrasien. Ces travaux ont eu pour objectif principal d'analyser l'impact des politiques commerciales ou d'ajustement structurel visant à réformer l'économie tunisienne en la rendant plus ouverte aux marchés internationaux. Pour une revue de ces modèles EGC, voir Cokburn et al. (2001).

10. Il est bien clair que le problème de gestion de l'eau en agriculture est plutôt dynamique en raison principalement de la variabilité climatique interannuelle et la réduction de ses risques par le recours à l'irrigation. Toutefois, l'introduction d'une telle dynamique ajouterait dans notre modèle EGC un degré de complexité supplémentaire d'autant plus que celui-ci est déjà très désagrégé afin de prendre en compte les effets intersectoriels des politiques de tarification d'eau 
d'irrigation. Dans ce travail, comme il a été décidé de privilégier ce dernier élément, il nous a paru nécessaire de laisser de côté la dimension temporelle de la gestion de l'eau en agriculture et de recourir seulement à un modèle EGC statique.

11. Cette forme fonctionnelle est non décroissante, continue et quasi concave en tout point. 12. Sur ce point, voir par exemple Burniaux (1987) pour une description détaillée.

12. . Sur ce point, voir par exemple Burniaux (1987) pour une description détaillée.

13. La gestion des besoins non agricoles en eau est principalement sous la responsabilité de la Société nationale d'exploitation et de distribution des eaux (SONEDE) qui est une entité autonome sur le plan financier mais se trouve sous la tutelle du ministère de l'Agriculture et des Ressources hydrauliques.

14. . On a considéré ici que l'offre de terre irriguée a une élasticité nulle et que celle de l'eau est infinie. Dans le modèle, la première est faible, la seconde est grande sans être infinie.

15. . On peut noter que la baisse de prix apporte à l'usager un gain de surplus égal à la somme des aires $A+s$, alors que le service de l'eau ne perd en recette que $A$.

16. . D'après le ministère de l'Agriculture et des Ressources hydrauliques, l'objectif préliminaire de la tarification de l'eau d'irrigation serait de couvrir $30 \%$ des coûts fixes liés à l'infrastructure hydraulique. Néanmoins, la couverture totale des coûts fixes a été simulée et les résultats sont de même signe mais de plus fortes amplitudes et confirment les conclusions de ce texte. Pour plus de détails, $c f$. Thabet (2003).

17. . TCM, TBP et $T B C$ désignent respectivement tarification au coût moyen, tarification « binôme personnalisée » et tarification «binôme classique ». MD : millions de dinars, sachant qu'en 1992, un dinar tunisien équivalait à un dollar américain.

18. . La production des agrumes de la région de Nabeul représente plus de $80 \%$ de la production totale du pays.

19. . Le mode de métayage le plus répandu dans l'agriculture tunisienne est le Khemmassa dans lequel le métayer est rémunéré par le cinquième de la production en volume.

20. . Dans la tarification binôme personnalisée, la taxe à la terre irriguée s'élève à $38 \%$ de la valeur locative d'un hectare de terre irriguée (égale à 1 à l'année de base).

21. . Pour plus de détails, voir Thabet (2003).

\section{RÉSUMÉS}

À l'aide d'un modèle d'équilibre général calculable centré sur le secteur agricole et agroalimentaire tunisien, nous comparons au système actuel de subvention trois méthodes de tarification de second rang de l'eau d'irrigation en termes d'efficacité et d'équité : i) une tarification au coût moyen, ii) une tarification binôme "classique», et iii) une tarification binôme « personnalisée » où l'abonnement est appliqué à l'hectare de terre irriguée. Les résultats montrent que le plus fort gain d'efficacité globale est obtenu par cette dernière. Si les pouvoirs publics ont pour objectif de faire contribuer les agriculteurs au financement de l'infrastructure hydraulique sans entraver l'irrigation, ni affecter la balance commerciale agricole et alimentaire, la tarification binôme personnalisée est l'instrument le plus adapté. Si par contre, l'objectif primordial est d'économiser l'eau, la tarification au coût moyen est la plus appropriée.

A computable general equilibrium model focused on Tunisian agriculture and agri-food sector was built to compare in terms of efficiency and equity, three alternative second best pricing 
methods of irrigation water, namely: i) an average cost pricing method, ii) a "classical" two-tier pricing method, and iii) a "personalized" two-tier pricing method where a fixed levy is applied per hectare of irrigated land. Results show that if the policy makers' aim is to increase farmers' contribution to the cost of hydraulic infrastructure without hindering irrigation nor affecting agricultural and food balance of trade, the "personalized" two-tier pricing method is the best instrument. If, on the contrary, the essential objective is to save water, average cost pricing is more appropriate.

INDEX

Code JEL Q18 - Agricultural Policy; Food Policy, Q25 - Water, Q15 - Land Ownership and Tenure; Land Reform; Land Use; Irrigation

Mots-clés : modèles EGC, réforme des prix de l'eau, tarification au coût moyen, tarification binôme

Keywords : average cost pricing, CGE models, two-tier pricing, water pricing reform

\section{AUTEURS}

\section{CHOKRI THABET}

École supérieure d'horticulture et d'élevage de Chott Mariem, Sousse, Tunisie

LOUIS-PASCAL MAHÉ

Département d'économie et de gestion, Agrocampus/ENSA de Rennes

\section{YVES SURRY}

Swedish University of Agricultural Sciences, Uppsala, Suède 